\title{
NOTA
}

\section{Perfil profesional de los agentes de empleo y desarrollo local en España}

\section{Joan Ramon Sanchis Palacio' \\ Amparo Melián Navarro}

Palabras clave: Desarrollo local, Agentes de Empleo y Desarrollo Local, inserción sociolaboral, políticas locales de empleo.

Key Words: Local Development, Agents of Employment and Local Development, SocialProfessional Integration, Local Employment Policies.

Mots clés: Développement local, Agents d’emploi et développement local, inclusion sociale et du travail, politiques locales d'emploi.

\section{Introducción}

Los Agentes de Empleo y Desarrollo Local (AEDLs) son participantes activos en la formulación e implementación de las políticas locales de empleo y en todos aquellos aspectos asociados al desarrollo local y cuya finalidad principal es la inserción sociolaboral de los diferentes colectivos que buscan integrarse en el mercado de trabajo.

${ }^{1}$ Profesor Titular de Universidad. Universitat de Valencia

${ }^{2}$ Catedrática de Escuela Universitaria. Universidad Miguel Hernández de Elche 
Dichos agentes desempeñan una labor de intermediación que se hace imprescindible en la actualidad para aproximar las iniciativas locales de empleo a aquellos colectivos más necesitados. Sin embargo, hasta hace muy poco tiempo, los profesionales del desarrollo local han pasado prácticamente desapercibidos, su trabajo está siendo escasamente reconocido en los ámbitos político, social y laboral y están siendo objeto de una legislación tanto estatal como autonómica que los condena a la temporalidad, la dependencia política y la insuficiencia de recursos para realizar eficientemente su trabajo.

La realización de un diagnóstico estratégico sobre la realidad del AEDL en España y para cada una de las comunidades autónomas que la constituyen, puede ayudar a comprender cuál es la situación actual del mismo y a proponer medidas que resuelvan en parte o en su totalidad las debilidades existentes.

En el sentido apuntado anteriormente, el presente trabajo tiene por objeto el estudio del perfil del AEDL en una triple dimensión: laboral, formativa y profesional. La metodología a seguir para la consecución de los objetivos expuestos consiste en la realización de un trabajo de campo durante el período comprendido entre el segundo semestre de 2006 y el primer semestre de 2007, para la explotación de un cuestionario dirigido a los AEDLs para la obtención de información sobre su perfil laboral, formativo y profesional. El objetivo del trabajo es un análisis descriptivo de la situación real de los AEDLS en España, sin pretensiones de análisis estadísticos más complejos que quizás puedan disfrazar la realidad en la que se mueve este colectivo. Consideramos que puede ser interesante un trabajo en el que se aporta información de primera mano obtenida de un estudio inédito realizado en España hasta ese momento sobre la realidad de los AEDLS en nuestro país.

El trabajo se ha estructurado en cuatro apartados diferentes, incluido este primero de introducción. En el segundo apartado se analizan los resultados obtenidos sobre el perfil de los AEDLs en los diferentes estudios empíricos realizados hasta el momento a partir de los cuales se formulan las hipótesis a contrastar en nuestro estudio. En el tercer apartado se procede a la presentación de los resultados obtenidos del estudio empírico realizado sobre la situación real actual del agente en relación con su perfil. En el cuarto y último apartado se presentan las conclusiones obtenidas del estudio realizado. El trabajo finaliza con la relación de fuentes bibliográficas utilizadas en el estudio. 


\section{La figura del AEDL: principales características de su perfil en España}

Los AEDLs llevan a cabo su actividad en organizaciones de desarrollo local, por lo que incluyen aspectos tan diversos como el asesoramiento, la formación, la prestación de servicios de información o actividades específicas como el Turismo Rural, entre otras (ADELA, 1991; Antuñano et al., 1993; Orero, 1993; Sanchis 2004; Calvo Palomares, 2009).

El análisis de su realidad se ha estudiado desde diferentes puntos de vista, entre los cuales se puede destacar la visión territorial (Allende Landa, 1987; Alburquerque, 1997; Camagni, 2003; Honrubia López, 2004; Mozas y Bernal, 2006; De Pablo y Uribe, 2009; Manrique de Lara, 2008), la visión económica y empresarial (Cruz Roche, 1984; Vázquez Barquero y Garofoli, 1995; Gazón, 1996; Contell García, 1996; Cachón Rodríguez, 1999; Giner Mansó, Muriel de los Reyes y Toledano Redondo, 1999; Alonso Santos y Méndez, 2000; Calvo Palomares, 2008), la visión cooperativa (Sola Sola y Pellicer, 1992; Costa Campí et al., 1993; OCDE, 1999; Sanchis, 2001) y la visión estratégica (Sanchis, 1999; Becattini, Costa Campí y Trullen, 2002, Tomás Carpi, 2008). También existe una literatura destacada sobre el papel del agente en el desarrollo local desde una perspectiva práctica, aspecto este en el cual se va a centrar el presente trabajo.

Mediante el análisis de los diferentes estudios empíricos realizados con anterioridad sobre el tema, los cuales quedan recogidos en la Tabla 1, se pueden obtener algunas conclusiones previas sobre la situación real de los agentes, que pueden contrastarse con los resultados obtenidos del estudio empírico realizado en el presente trabajo.

En el primer estudio referido a las agencias de desarrollo local de Canarias ya se demostraban algunas de las cuestiones que en estudios posteriores se constatan con mayor rotundidad: la temporalidad de los agentes y la falta de medios técnicos y humanos de las agencias, principalmente. En él, además, se analiza el protagonismo de las empresas de la economía social en el desarrollo local, demostrando su importancia. 


\section{TABLA I. Principales estudios empíricos publicados en España sobre los AEDLs}

\begin{tabular}{|l|l|c|l|l|}
\hline Estudios & \multicolumn{1}{|c|}{ Ámbito } & Período & \multicolumn{1}{|c|}{ Objetivos } & Metodología \\
\hline $\begin{array}{l}\text { Medina, Rodríguez y } \\
\text { Fuentes (1996) }\end{array}$ & Canarias & $1992-94$ & $\begin{array}{l}\text { Funciones de las agencias } \\
\text { y perfil del agente }\end{array}$ & Cuestionario \\
\hline $\begin{array}{l}\text { Sanchis y Cantarero } \\
\text { (1999) }\end{array}$ & $\begin{array}{l}\text { Comunidad } \\
\text { Valenciana }\end{array}$ & 1998 & $\begin{array}{l}\text { Entornoy perfil del agente } \\
\text { y características servicio }\end{array}$ & Cuestionario \\
\hline FOREM (2000) & Estatal & $\begin{array}{l}\text { Perfil laboral, profesional } \\
\text { y formativo del agente } \\
\text { Casos prácticos }\end{array}$ & $\begin{array}{l}\text { Cualitativa: en- } \\
\text { trevistas y gru- } \\
\text { pos discusión }\end{array}$ \\
\hline $\begin{array}{l}\text { Campos y Cantarero } \\
\text { (2002) }\end{array}$ & $2000-05$ & $\begin{array}{l}\text { Entornoy perfil del agente } \\
\text { y características servicio }\end{array}$ & Cuestionario \\
$\begin{array}{l}\text { Sanchis, Cantarero } \\
\text { y Campos (2003a y } \\
\text { 2003b) } \\
\begin{array}{l}\text { Sanchis y Campos } \\
\text { (2002 y 2005); } \\
\text { Sanchis (2006) }\end{array}\end{array}$ Estatal & 2003 & $\begin{array}{l}\text { Situación del Desarrollo } \\
\text { Local }\end{array}$ & Cuestionario \\
\hline \begin{tabular}{l} 
Aprodel (2004) \\
\hline
\end{tabular}
\end{tabular}

Fuente: elaboración propia

El trabajo publicado por FOREM (2000) es un estudio empírico sobre las necesidades formativas de los agentes de desarrollo local y figuras profesionales afines y recoge conclusiones relacionadas con el perfil laboral, profesional y formativo de los agentes (edad, formación, género, etc.), así como otros aspectos relacionados con éste, tales como la falta de medios, la indefinición del puesto y el desconocimiento por parte de la institución (ayuntamientos); asimismo destaca cuestiones como la temporalidad de sus contratos, lo que dificulta su continuidad. El informe señala también su incierto futuro a medio plazo y la relevancia de la intervención de los políticos en las iniciativas implementadas, así como la falta de coordinación en las actividades.

En los trabajos de Sanchis y Cantarero (1999), referido a la Comunidad Valenciana, y de Sanchis y Campos (2002 y 2005), Campos, Cantarero y Sanchis (2002) y Sanchis, Cantarero y Campos (2003a y 2003b), Sanchis (2006), referidos al 
conjunto estatal, se analiza, además del perfil laboral, formativo y profesional de los agentes en España, las características del servicio que éstos ofrecen, obteniendo conclusiones que coinciden con las apuntadas en el estudio de FOREM (2000). También se obtienen conclusiones relativas a aspectos relacionados con el servicio del agente (su trabajo se centra más en los aspectos técnicos y administrativos que en los realmente innovadores y creativos; los colectivos con los que mayoritariamente se trabaja y los proyectos que gestionan fundamentalmente). También se destacan características importantes en la realización del trabajo de estas figuras (capacidad de comunicación, de visión general, de selección de información y de gestión).

Por último, en el trabajo realizado por la Asociación de Profesionales del Desarrollo Local de Madrid (Aprodel, 2004), además de analizar el perfil del agente, se realiza un estudio sobre el panorama general del desarrollo local, concluyendo que las políticas de desarrollo local llevadas a cabo (en Madrid) se limitan simplemente a definir unas líneas generales, lo cual es insuficiente pues la aplicación de las nuevas tecnologías de la información y la elaboración de una normativa específica sobre el desarrollo local podrían ayudar de manera significativa a mejorar la formulación de estas políticas.

Todos los trabajos empíricos recogidos en la Tabla 1 coinciden en señalar que una de las características determinantes en la función del AEDL y que define su perfil profesional es la temporalidad del puesto ${ }^{3}$, lo cual unido a su escaso reconocimiento por parte de la Administración Pública y de la sociedad en general, hace que sus expectativas laborales sean bajas (temor permanente a la pérdida del puesto y escasa valoración profesional). La temporalidad del agente viene dada por el hecho que su continuidad en la agencia depende de las subvenciones del INEM o del organismo autonómico competente en materia de empleo (limitadas al $80 \%$ del coste total y a un tiempo máximo de cuatro años no prorrogable) o con cargo a un proyecto limitado en el tiempo (generalmente de la Unión Europea) y a la vinculación del puesto y del trabajo de desarrollo local a un programa político (gobierno municipal principalmente y en menor medida mancomunidad o diputación provincial).

\footnotetext{
${ }^{3}$ Conviene resaltar la posible conexión entre la temporalidad del puesto y el que el perfil del agente sea el de una mujer joven recién titulada (colectivo con alto riesgo de exclusión laboral). La Orden de 15 de julio de 1999 del Ministerio de Trabajo y Asuntos Sociales sobre contratación de los AEDLs establece que para poder participar en los procesos de selección y contratación se exige estar desempleado y con titulación universitaria (diplomado o licenciado), además de valorar la experiencia profesional y los conocimientos extraacadémicos adquiridos en cursos monográficos de desarrollo local o promoción de proyectos de empleo.
} 
En definitiva, según los estudios empíricos realizados y de acuerdo con los planteamientos teóricos recogidos en los trabajos más relevantes publicados sobre el Desarrollo Local (Vázquez Barquero, 1993, 1996a, 1996b, 1998; Rodríguez Gutiérrez, 1999; Martínez Puche, 2000; Pérez Ramírez y Carrillo Benito, 2001; Rodríguez Álvarez, 2001; González Carmona, 2007; Calvo Palomares, 2009), se formulan las siguientes hipótesis referidas a cada uno de los tres aspectos analizados en el estudio empírico realizado: el perfil laboral, el perfil formativo y el perfil profesional.

El perfil laboral del AEDL viene determinado, tal como se desprende de los estudios empíricos analizados anteriormente, por aspectos tales como la edad, el género, su experiencia laboral previa, los motivos por los cuales se presenta al puesto, el tipo de contratación y su grado de autonomía y de satisfacción en el puesto. Las hipótesis que se plantean en este sentido son cuatro:

Hipótesis 1: Persona joven, en su mayoría mujer, con escasa experiencia en temas de desarrollo local y motivación negativa (salida del desempleo).

Hipótesis 2: El puesto de AEDL tiene una gran dependencia de los poderes políticos y de subvenciones temporales y una elevada temporalidad.

Hipótesis 3: El grado de autonomía del AEDL en la fijación de objetivos y de acciones y en su ejecución es medio-bajo y sus expectativas inciertas al depender en gran medida de decisiones políticas y de subvenciones públicas.

Hipótesis 4: El grado de satisfacción del AEDL es mayor en el ambiente laboral, las funciones realizadas y las responsabilidad asumidas que en salarios, expectativas de futuro y reconocimiento social.

El perfil formativo del AEDL viene determinado, según los estudios empíricos analizados anteriormente, por aspectos relacionados con su formación teórica, sus carencias y necesidades formativas, los procesos de mejora formativa realizados y su experiencia profesional. En este sentido, se plantea la siguiente hipótesis:

Hipótesis 5: Los AEDLs, en su mayoría titulados en Derecho y en menor medida en titulaciones relacionadas con la empresa, tienen importantes necesidades formativas en temas específicos de desarrollo local y en aspectos relacionados con la economía y la empresa por la importancia del carácter multidisciplinar de sus acciones y por la importancia que tiene la experiencia profesional en el desarrollo local, por lo que existe una importante oferta formativa en este campo que los agentes suelen aprovechar. 
Por último, el perfil profesional del AEDL viene dado, en función de los estudios empíricos analizados en apartados anteriores, por aspectos relacionados con sus recursos y capacidades profesionales y por las funciones que realizan y actividades y colectivos a los cuales se dirigen. La hipótesis que se formula desde esta perspectiva es:

Hipótesis 6: La profesionalización del AEDL requiere de la capacidad de comunicación con los colectivos a los cuales se dirige y con la capacidad de gestión y control de las acciones llevadas a cabo.

\section{Perfil real del AEDL: resultados del estudio empírico.}

El trabajo de investigación que proponemos, realizado entre el segundo semestre de 2006 y el primer semestre de 2007, va referido al conjunto del Estado español a la vez que permite su desagregación por comunidades autónomas, por lo que el ámbito de este trabajo es superior al de los otros estudios empíricos realizados hasta la fecha y recogidos en la Tabla 1. El objeto de estudio del trabajo es el agente de inserción (su papel en el desarrollo local) en una concepción amplia de dicho término, por lo que incluye no solo a los agentes que realizan su trabajo en las Administraciones Públicas de ámbito local (Ayuntamientos), supralocal (Consorcios), comarcal (Oficinas Leader y Proder, Mancomunidades), provincial (diputaciones, UPDs, CEEls) y autonómico (organismos autonómicos), sino también a los pertenecientes a entidades privadas con y sin ánimo de lucro (universidades, sindicatos, asociaciones empresariales, cámaras de comercio, fundaciones y asociaciones, etc.). En este sentido, el estudio engloba a una realidad muy heterogénea y amplia de profesionales del desarrollo local superior a la incluida en otros estudios ya realizados.

El plan de trabajo se estructura en cuatro etapas o fases diferentes: en primer lugar, se ha elaborado un directorio nacional de agentes por Comunidades Autónomas, que ha servido de población muestral para la realización del estudio empírico ${ }^{4}$; en segundo lugar, se ha elaborado un cuestionario que incluye variables diversas determinantes del perfil laboral, formativo y profesional de los AEDLs y que ha sido

\footnotetext{
${ }^{4}$ El directorio de agentes de España elaborado por nosotros y utilizado para la realización de la presente investigación está incluido en una base de datos con formato Access, si bien por limitaciones de espacio no se ha incluido en el presente trabajo.
} 
enviado al total de la población censada ${ }^{5}$; en tercer lugar, se ha realizado el trabajo de campo consistente en el envío del cuestionario al conjunto de la población censada mediante correo postal y/ o correo electrónico durante el primer trimestre del año 2006; y, por último, en cuarto lugar se ha procedido a la tabulación y tratamiento estadístico de los datos obtenidos del trabajo de campo (programa informático SPSS versión 8.0.) mediante la realización de un análisis descriptivo a través de un recuento de frecuencias para cada una de las variables del cuestionario, mostrando así la situación real de cada grupo de variables. Con la información obtenida de la explotación de los datos del cuestionario, se ha realizado una valoración final de los resultados así como la presentación de las conclusiones de la investigación realizada durante el segundo trimestre del año 2007.

La muestra utilizada en la investigación ha sido de 3.469 agentes (que se corresponde con el total de la población censada), de la cual se han obtenido 481 respuestas (el $13,87 \%$ de la muestra), tal como aparece en la Tabla 2. A partir del número de respuestas obtenidas a nivel estatal, se ha obtenido un margen de error inferior $a+-6 \%$ para un nivel de confianza del $95,5 \%$, por lo que nos encontramos ante un estudio significativo desde el punto de vista estadístico.

TABLA 2. Índice de respuesta

\begin{tabular}{|l|r|r|r|c|}
\hline \multirow{2}{*}{ Comunidad Autónoma } & \multicolumn{2}{|c|}{ Población censada } & \multicolumn{2}{c|}{ Respuesta } \\
\cline { 2 - 5 } & Núm. & $\%$ & Núm. & $\%$ \\
\hline Andalucía & 1.138 & 32,80 & 54 & 4,75 \\
\hline Aragón & 89 & 2,57 & 22 & 24,72 \\
\hline Asturias & 70 & 2,02 & 18 & 25,71 \\
\hline Baleares & 24 & 0,69 & 4 & 16,67 \\
\hline Canarias & 219 & 6,31 & 20 & 9,13 \\
\hline Cantabria & 40 & 1,15 & 12 & 30,0 \\
\hline
\end{tabular}

${ }^{5}$ El cuestionario es semiestructurado, incluyendo en el mismo, tanto cuestiones abiertas (en las que se solicita información puntual sobre la variables) como cuestiones cerradas (en las que se solicita al encuestado que señale la opción correcta entre múltiples alternativas). Las variables abiertas incluyen: variables dicotómicas (sí/ no), variables con múltiples opciones en las que el encuestado ha de dar o bien una única respuesta o bien múltiples respuestas (en este caso la suma de los porcentajes obtenidos será superior a 100) y variables que se miden mediante la escala de Likert con valores comprendidos entre 1 y 5. 


\begin{tabular}{|l|r|r|r|r|}
\hline Castilla-La Mancha & 267 & 7,70 & 55 & 20,60 \\
\hline Castilla-León & 225 & 6,49 & 35 & 15,55 \\
\hline Cataluña & 263 & 7,58 & 28 & 10,65 \\
\hline Euskadi & 40 & 1,15 & 18 & 45,00 \\
\hline Extremadura & 206 & 5,94 & 17 & 8,25 \\
\hline Galicia & 347 & 10,00 & 114 & 32,85 \\
\hline La Rioja & 21 & 0,61 & 9 & 42,86 \\
\hline Madrid & 79 & 2,28 & 9 & 11,39 \\
\hline Murcia & 74 & 2,13 & 13 & 17,57 \\
\hline Navarra & 36 & 1,04 & 5 & 13,88 \\
\hline C. Valenciana & 307 & 8,85 & 47 & 15,31 \\
\hline Ceuta y Melilla & 24 & 0,69 & 1 & 4,17 \\
\hline España & $\mathbf{3 . 4 6 9}$ & $\mathbf{1 0 0 , 0 0}$ & $\mathbf{4 8 1}$ & 13,87 \\
\hline
\end{tabular}

Fuente: elaboración propia

En definitiva, el análisis de resultados se realizará tanto referido al conjunto estatal como por Comunidades Autónomas. No se incluirá en el análisis, sin embargo, el caso de las ciudades autónomas de Ceuta y Melilla al haberse obtenido un único cuestionario.

\section{I. Perfil laboral del AEDL}

El perfil laboral del agente viene definido por las Tablas 3, 4, 5 y 6 recogidas en el anexo. De la Tabla 3 se deduce que la denominación predominante es la de Agente de Empleo y Desarrollo Local (en algo más de la mitad de los casos) y en menor medida la de Técnico Local o Promotor de Empleo (en el 25\% de los casos). Esto es así por cuanto es la misma denominación oficial de esta figura del desarrollo local que se utiliza en la Orden ministerial de 1999 de fomento del Desarrollo Local e impulso de los proyectos y empresas calificadas como l+E.

No obstante, existen también otras denominaciones que en determinadas comunidades autónomas tienen un peso relevante. Así, por ejemplo, la denominación de técnico local, que a nivel estatal representa el 13\% del total, es la mayoritaria en Galicia (45\%) y adquiere un peso significativo en Murcia (31\%). También la 
denominación de promotor de empleo representa en todo el Estado un porcentaje destacado del $12 \%$, es la denominación mayoritaria en Murcia (46\%) y tiene un peso significativo en Andalucía (20\%), Baleares (25\%), Euskadi (22\%) y Galicia (37\%). La denominación de agente de desarrollo local, con un $10 \%$ de peso a nivel estatal, es mayoritaria en Asturias (44\%) y tiene una importancia en peso destacada en Baleares (25\%), Cantabria (42\%) y Castilla León (23\%). Por último, podemos señalar que la denominación de agente de desarrollo rural, que a nivel estatal tiene un peso muy reducido de casi el $2 \%$, en tres comunidades llega a tener un peso significativo: Canarias (15\%), La Rioja (22\%) y Navarra (20\%).

El AEDL es en su gran mayoría mujer (más del $60 \%$ del total) de entre 25 y 35 años de edad (más del $60 \%$ de los casos), con una antigüedad en el puesto no superior a los tres años (más del $60 \%$ de los casos) y cuya motivación principal ha sido una motivación de tipo personal (57\%) y en menor medida para salir de una situación de desempleo (39\%). Se trata, por tanto, de una mujer joven con pocos años en el puesto que busca en el puesto de agente la posibilidad de desarrollarse profesionalmente y de insertarse en el mercado laboral. La media de antigüedad en el puesto es de 3,7 años.

Las comunidades de Castilla-La Mancha y Madrid poseen los agentes más jóvenes de España, mientras que las plantillas de mayor edad se sitúan en Navarra y Cantabria. Con respecto a la antigüedad en el puesto, el valor medio más alto (mayor antigüedad en el puesto) se da en Navarra (8 años) y Asturias (5 años) y el valor medio más bajo (menor antigüedad en el puesto) se da en La Rioja (2 años), Murcia ( 2 años) y Aragón (casi 3 años). Por último, en relación con los motivos por los cuales han decidido ocupar el puesto de agente también existen diferencias por comunidades autónomas: salir de una situación de desempleo es el motivo principal para los agentes de Canarias y Castilla-La Mancha y tener experiencia anterior es el motivo mayoritario en Asturias, Cantabria y Madrid; en el resto de comunidades autónomas, el motivo principal es el mismo que para la media del Estado, es decir, la motivación personal.

De esta forma se demuestra la veracidad de la Hipótesis 1 según la cual el AEDL es un titulado universitario joven, en su mayoría mujer, con escasa experiencia en temas de desarrollo local y motivación negativa (salida del desempleo).

La Tabla 4 describe las características relacionadas con la contratación de los agentes. En este sentido, se puede señalar que el agente es un contratado temporal por obra o servicio (o en el mejor de los casos laboral temporal), cuyo puesto depende de una subvención, la cual procede mayoritariamente de la Administración 
Autonómica (Consejería de Trabajo) y en mucha menor medida de la Administración Local o del INEM. El agente es contratado en la mayoría de los casos por el ayuntamiento y el proceso de selección incluye como principales ejercicios la valoración del curriculum vitae y la entrevista personal.

Las comunidades autónomas con mayor porcentaje de contratos fijos son Andalucía y Baleares con unos porcentajes destacados de contratados laborales (20\% y $25 \%$ respectivamente) y Cataluña y $C$. Valenciana donde el peso de los contratados fijos funcionarios es significativo ( $14 \%$ y $17 \%$ respectivamente). La entidad financiadora, que a nivel estatal es con diferencia la consejería u organismo autonómico correspondiente, varía según comunidades: el INEM es la entidad financiadora mayoritaria en Andalucía (59\%), Castilla-La Mancha (60\%) y Euskadi (86\%), aunque en muchas otras comunidades su peso es insignificante o incluso inexistente (Baleares, Cataluña, Galicia, Madrid, Murcia y Navarra). Esto tiene que ver con el momento en que se han producido las transferencias en materia de empleo a las autonomías; precisamente son Andalucía y Castilla-La Mancha las últimas comunidades que recibieron dichas transferencias (a mediados del 2003), mientras que Euskadi aún no las ha recibido. Es de esperar que el peso del INEM en la financiación de la contratación de los agentes se vaya reduciendo de manera significativa durante los próximos años, a la vez que el peso de las consejerías $u$ organismos autonómicos correspondientes vaya aumentando. Las diputaciones provinciales juegan un papel financiador importante en Castilla-La Mancha (20\%) y Castilla-León (13\%), aunque en muchas comunidades no tienen ninguna relevancia. Por su parte, el papel financiador de las mancomunidades destaca en Baleares (25\%). Conviene señalar que en casi todas las comunidades, la financiación del puesto de agente es compartida por varias de las entidades mencionadas.

Por otra parte, la entidad que realiza la contratación de los agentes son mayoritariamente las administraciones locales, excepto en Baleares (0\%) y Galicia (21\%). En Baleares, son en porcentajes similares la consejería, el INEM, las mancomunidades e incluso empresas privadas sin ánimo de lucro (fundaciones). En Galicia, la entidad contratante es la consejería de empleo. En Madrid destaca el peso significativo del INEM (43\%) y en Canarias el de las mancomunidades (40\%). Las diputaciones provinciales también destinan sus esfuerzos a la contratación de agentes, sobre todo en Castilla-León (29\%) y Castilla-La Mancha (36\%). Además de las entidades mencionadas, en algunas comunidades autónomas intervienen otras instituciones como las universidades o las federaciones y confederaciones de empresarios (en Galicia el peso de estas entidades es significativo). También en este caso, la contratación se produce entre varias entidades conjuntamente. 
Por último, los procesos de selección para la contratación de los agentes varían de unas comunidades a otras. Por ejemplo, la realización de pruebas escritas, que a nivel estatal tiene mucha menor significación que otros procesos, en Euskadi, La Rioja y Navarra son utilizadas en un $80 \%$ de los casos aproximadamente y en las dos Castillas se aproximan al 50\% de los casos. La presentación de un proyecto, que a nivel estatal representa el 28\%, en Extremadura representa el $77 \%$ y en la C. Valenciana el $68 \%$. No obstante, en todas las comunidades autónomas tienen una gran relevancia como pruebas de selección la valoración del currriculum vitae y la realización de entrevistas personales, por ese orden, si bien también se aprecian algunas diferencias aunque poco significativas. Conviene señalar que en todos los casos, el proceso de selección consiste en una valoración conjunta a partir de los diferentes aspectos contemplados.

De esta forma se demuestra la veracidad de la Hipótesis 2 según la cual el puesto de AEDL tiene una gran dependencia de los poderes políticos y de subvenciones temporales y una elevada temporalidad.

El grado de autonomía en el trabajo de agente y las expectativas futuras en el puesto quedan reflejados en la Tabla 5 . Como se puede apreciar, el grado de autonomía del agente en la realización de las actividades relacionadas con la planificación y ejecución de su puesto es parcial en la gran mayoría de los casos y es mayor en la ejecución de las acciones que en la fijación de dichas acciones y de los objetivos a alcanzar. El nivel de autonomía alcanzado por el agente en su puesto depende en gran medida de la organización del trabajo realizado y en mucha menor medida de la jerarquía y de la antigüedad.

Por su parte, las expectativas de mantenerse en el puesto no son positivas, ya que casi la mitad de los agentes manifiestan desconocer qué va a pasar en el futuro con su puesto, y ello lo atribuyen a la subvención del puesto que ocupan en la inmensa mayoría de los casos y a una decisión políitica; en cambio, el alcanzar o no los resultados previstos (eficacia de la acción) es un factor que apenas tiene importancia en dichas expectativas.

El grado de autonomía de los agentes en la realización de tareas como la fijación de objetivos, la definición de acciones a llevar a cabo y la ejecución de dichas acciones es muy similar en todas las comunidades autónomas, si bien existen algunas diferencias significativas. Respecto a la autonomía en la fijación de objetivos, la comunidad con mayor autonomía es Navarra (el gado total de autonomía alcanza un porcentaje del 60\%), seguida de Cantabria y Extremadura (alrededor del $40 \%$ en ambos casos). Respecto al nivel de autonomía en la fijación de acciones, son 
también Navarra y Extremadura las que presentan mayor autonomía, junto con Canarias (alrededor del $40 \%$ de autonomía total). Por último, respecto a la autonomía en la ejecución de las acciones no existen diferencias significativas, pues en todas las comunidades el nivel de autonomía es alto. En cuanto a los factores que determinan dichos niveles de autonomía, el factor relevante en casi todas las comunidades autónomas, al igual que a nivel estatal, es la organización del trabajo. Sólo en el caso de Madrid se observa un peso igual para el factor relacionado con la antigüedad en el puesto (43\%), factor que también es significativo en Canarias $(40 \%)$ y en Navarra (40\%).

En segundo lugar, podemos afirmar que el mayor desconocimiento sobre las expectativas en el puesto de agente se produce en Baleares (75\% de los casos), en Castilla-La Mancha (56\%) y en Canarias (55\%). Por el contrario, las mayores expectativas de consolidación del puesto se dan en Navarra (80\%), Cantabria (58\%), Madrid (57\%) y Euskadi (56\%). En cuanto a los factores que condicionan dichas expectativas (decisión política, dependencia de una subvención y en mucha menor medida el alcanzar los resultados previstos), hay que señalar que en las únicas comunidades donde adquiere un peso significativo el factor técnico (cumplir los objetivos previstos o alcanzar resultados deseados) es en Navarra, Euskadi y Cantabria (entre el $40 \%$ y el $50 \%$ ), precisamente las comunidades donde las expectativas de consolidación son mayores. En cambio, los factores políticos (decisión política y dependencia de una subvención) tienen mayor peso en Canarias.

De esta forma se demuestra la veracidad de la Hipótesis 3 según la cual el grado de autonomía del AEDL en la fijación de objetivos y de acciones y en su ejecución es medio-bajo y sus expectativas inciertas al depender en gran medida de decisiones políticas y de subvenciones públicas.

Por último, con el propósito de obtener un perfil laboral del agente lo más completo posible, en la Tabla 6 se analiza el grado de satisfacción del agente en diversos aspectos de su trabajo: las funciones realizadas, la responsabilidad ejercida, el salario, el ambiente laboral, las perspectivas de futuro, los resultados obtenidos y el reconocimiento social alcanzado. En este sentido, se observa que los mayores niveles de satisfacción se dan en las funciones realizadas, en el ambiente laboral, en los resultados obtenidos y en la responsabilidad ejercida, es decir, en aquellos aspectos asociados con un trabajo con gran autonomía y de gran satisfacción profesional. Sin embargo, los menores niveles de satisfacción se dan en las perspectivas de futuro (el futuro del agente es incierto al depender de una subvención y de una decisión política), en el salario (normalmente salarios bajos unido a contratos temporales) y en el reconocimiento social (desconocimiento del 
puesto y el papel que realiza por parte de la sociedad y escaso reconocimiento político a su labor).

De esta forma se demuestra la veracidad de la Hipótesis 4 según la cual el grado de satisfacción del AEDL es mayor en el ambiente laboral, las funciones realizadas y las responsabilidad asumidas que en salarios, expectativas de futuro y reconocimiento social.

\subsection{Perfil formativo del AEDL}

El perfil formativo del agente se resume en las Tablas 7, 8, 9 y 10 del anexo, en las que se analiza su experiencia laboral, grado de titulación, nivel de formación complementaria y carencias formativas y necesidades de conocimientos para realizar su trabajo. En este sentido, un primer aspecto a señalar es la experiencia laboral previa que poseen los agentes (las tres cuartas partes tenían experiencia laboral, algo más de la mitad tenían experiencia relacionada con el trabajo de agente y el $40 \%$ había trabajado ya de agente en ocasiones anteriores) y la utilidad de dicha experiencia (el 90\% de los agentes así lo manifiestan) (Tabla 7). Los mayores porcentajes de experiencia del agente se dan en Asturias, La Rioja y Madrid, y los menores porcentajes se dan en Baleares y Canarias.

Según la Tabla 8, el 99,2\% de los agentes son titulados universitarios y las titulaciones predominantes son las jurídicas y las relacionadas con el mundo de la empresa y la economía: el 20\% de los agentes son licenciados en Derecho, el 17\% son licenciados en Empresariales, el $11 \%$ diplomados en Relaciones laborales y el $10 \%$ licenciados en Economía. No obstante, existen agentes con titulaciones muy diversas que no tienen relación directa con la empresa o la economía (Pedagogía, Ingeniería Técnica Industrial, Biología, Ciencias Políticas, Magisterio, Periodismo o Documentación, entre otras). Conviene resaltar que el $78 \%$ de los agentes consideran que su formación es adecuada, lo cual va unido al hecho que más del $90 \%$ han realizado cursos de formación complementaria y que el $85 \%$ de las agencias fomentan la realización de dichos cursos.

Las comunidades con mayor número de agentes sin titulación universitaria son Galicia $(23 \%)$ y en menor medida Murcia $(8,3 \%)$ y Extremadura $(6,3 \%)$. Respecto a las titulaciones predominantes señalar que la licenciatura en Derecho es la mayoritaria a nivel estatal y en las comunidades de Aragón, Baleares, Castilla-La Mancha y La Rioja alcanzan porcentajes superiores al $30 \%$ del total. No obstante, hay otras titulaciones que tienen un peso significativo en determinadas comunidades. Así, 
los agentes con la licenciatura de Empresariales (la segunda con más peso a nivel estatal) son mayoritarios en Castilla-León (20\%) y Euskadi (55,6\%), y en Galicia y Andalucía tienen el mismo peso que los licenciados en Derecho $(21,1 \%$ y $24,1 \%$ respectivamente). La diplomatura de Relaciones Laborales (la tercera con más peso a nivel estatal), es la mayoritaria en Canarias (30\%) y en Murcia (33,3\%). La licenciatura en Geografía e Historia, con un peso por debajo del $10 \%$ a nivel estatal, es la mayoritaria en Asturias (27,7\%), Cantabria (50,1\%), Cataluña (25\%) y Navarra (40\%). Otras titulaciones con cierta relevancia en determinadas comunidades son la diplomatura de Trabajo Social en Murcia (25\%), la licenciatura de Sociología en Madrid (16,7\%), la licenciatura de Biología en Baleares (25\%) o la licenciatura de Pedagogía en Madrid (16,5\%), entre otras.

Merece destacar que los agentes de Aragón y de Madrid son los que menor formación apropiada para su trabajo tienen según ellos y que las comunidades que más se procupan por la formación de sus agentes son Galicia (97,4\%), Extremadura $(94,1 \%)$ y Murcia (92,3\%). Por último, los agentes que más cursos de formación realizan son los de Asturias, Madrid y Murcia (100\% en los tres casos) y los que menos los de Baleares (75\%) y Navarra (80\%).

Los tipos de cursos de formación realizados por los agentes son muy diversos, según muestra la Tabla 9, aunque destacan aquellos cursos cuyos contenidos están relacionados con el desarrollo local (75\% de los agentes), la economía y la empresa (48\%), la gestión de proyectos $(49 \%)$, la informática $(52 \%)$ y la gestión de recursos humanos $(22 \%)$, por ser éstas las herramientas más utilizadas en su trabajo como agentes. Las entidades a las que han acudido los agentes para la realización de dichos cursos son, además de la propia entidad a la cual pertenecen $(28 \%)$, la universidad (34\%), una entidad privada (28\%), el INEM (19\%), las diputaciones provinciales (17\%) y las consejerías (15\%). No obstante, la oferta de entidades es muy amplia.

En relación con los diferentes tipos de cursos formativos realizados por los agentes, hay que señalar que la mayor parte de las comunidades autónomas incluyen cursos en desarrollo local, economía y empresa, gestión de proyectos, informática y gestión de los RR.HH. La Rioja es la comunidad que mayor variedad de cursos formativos tiene, pues además de los señalados también incluye cursos más específicos sobre la Unión Europea, la orientación laboral, la inserción sociolaboral, la prevención de riesgos laborales y las políticas de igualdad de género en porcentajes próximos al 13\%. También se han realizado cursos sobre turismo en Asturias y Castilla-La Mancha y de idiomas en Euskadi. Los cursos de orientación laboral son especialmente importantes en Murcia (30,8\%) y Navarra (25\%). 
Las entidades dedicadas a la realización de cursos de formación para los agentes son principalmente la universidad y la propia entidad, aunque su peso y la existencia de otras clases de entidades formadoras varía según comunidades autónomas. Así, el mayoritario papel formador de los agentes por parte de las universidades destaca en Valencia (60\%) y Murcia (53,8\%). En cambio, la propia entidad a la que pertenece el agente asume el liderazgo de la formación en comunidades como Baleares $(66,7 \%)$ y Navarra (75\%). Por su parte, otras entidades tienen también un peso relevante en la formación de los agentes en determinadas comunidades: el INEM y la diputación provincial en Extremadura (56,3\% cada una), las entidades privadas (academias, centros privados de formación) en Andalucía (42,9\%), Baleares $(66,7 \%)$, Cantabria (72,7\%), Euskadi (40\%) y Madrid (57,1\%), la consejería de empleo en Galicia $(36,6 \%$ ) y la Federación Española de Municipios y Provincias (FEMP) en Madrid $(28,6 \%)$. Por último, existen otras entidades con menor peso dedicadas también a la formación de los agentes como la UNED $133,3 \%$ en la C. Valenciana), las cámaras de comercio (10\% en Aragón) y los sindicatos (6,9\% en Castilla-León).

Según la Tabla 10 se puede observar que las carencias mostradas por los agentes son mínimas, salvo en el caso de derecho y de economía donde se aprecia un porcentaje algo superior de carencia. El reducido grado de carencias es explicado por la importancia de la formación complementaria desarrollada por los agentes y que se ha analizado en la Tabla 9. Lo mismo se observa respecto a las necesidades de conocimientos; éstas son mínimas y sólo se aprecia un mayor porcentaje de necesidades en conocimientos relacionados con la economía y la empresa.

Por comunidades autónomas, los menores porcentajes de carencias en general se dan en Euskadi y los mayores en Baleares, si bien las diferencias no son significativas entre comunidades autónomas. Lo mismo sucede con respecto a las necesidades de conocimientos.

De esta forma se demuestra la veracidad de la Hipótesis 5 según la cual los AEDLs tienen importantes necesidades formativas en temas específicos de desarrollo local y en aspectos relacionados con la economía y la empresa por la importancia del carácter multidisciplinar de sus acciones y por la importancia que tiene la experiencia profesional en el desarrollo local. 


\subsection{Perfil profesional del AEDL}

El perfil profesional del agente se obtiene del estudio de las Tablas 11, 12 y 13 del anexo al analizar las características que son necesarias para realizar su trabajo, los mecanismos de control que utilizan, las relaciones que mantienen con otros agentes y su reconocimiento profesional por parte de los directores de las agencias, de los políticos, de los colectivos con los cuales trabajan y de la comunidad local en la cual realizan sus funciones.

De la Tabla 11 se deduce que todas las características expuestas son necesarias para realizar el trabajo de agente, si bien las más destacadas son la capacidad de comunicación, la visión general, la capacidad para seleccionar información y la capacidad de gestión.

La práctica totalidad de los agentes realizan una memoria de actividades donde se incluyen las acciones emprendidas y en una gran mayoría realizan también una evaluación del trabajo desempeñado, tal como muestra la Tabla 12. Por otra parte, la inmensa mayoría de los agentes mantienen relaciones con agentes de otras agencias y entidades, aunque dichas relaciones no suelen estar formalizadas sino que obedecen en la gran mayoría de los casos a contactos esporádicos (88\%) y a la celebración de reuniones puntuales (72\%). Sin embargo, es mucho menos habitual pertenecer a una asociación de profesionales $(31 \%)$ o a un grupo formal de trabajo (32\%).

Los mecanismos de control del trabajo del agente (memoria de actividades y sistema de evaluación del trabajo) son mayores en comunidades como Aragón, Cantabria, Cataluña, Euskadi, Extremadura, Galicia y Navarra. En cambio, son inferiores a la media estatal en Baleares y en Madrid. Las relaciones entre agentes son mayoritarias en todas las comunidades (por encima del $90 \%$ del total), si bien hay dos comunidades, Euskadi y Madrid, que muestran un peso inferior al $90 \%$ pero por encima del $80 \%$. Los tipos de relaciones más comunes son los contactos esporádicos y las reuniones puntuales, salvo en el caso de Baleares, donde tienen mayor peso la participación en grupos formales e informales de trabajo. No obstante, algunas comunidades utilizan además otros tipos de relaciones como la pertenencia a asociaciones (Asturias, Castilla-La Mancha, Castilla-León, Euskadi, Extremadura, Madrid, Navarra y C. Valenciana), la participación en grupos formales de trabajo (Euskadi, Murcia y Navarra) y la participación en grupos informales (Extremadura).

Por último, el reconocimiento del trabajo realizado por el agente es elevado por 
parte de los colectivos con los cuales trabajan y por parte de los directores de las agencias en las cuales trabajan (por encima del $60 \%$ si se consideran los valores 4 y 5 de la escala de Likert), y algo menor por parte de los políticos y de la comunidad local (alrededor del 50\%). Por comunidades autónomas, el mayor reconocimiento del trabajo del agente por parte del director se da en Madrid y el menor en Extremadura; el reconocimiento político es mayor en Murcia, al igual que el de los colectivos; y el reconocimiento de la comunidad local es mayor en La Rioja y menor en Baleares y Navarra.

De esta forma se demuestra la veracidad de la Hipótesis 6 según la cual la profesionalización del AEDL requiere de la capacidad de comunicación con los colectivos a los cuales se dirige y con la capacidad de gestión y control de las acciones llevadas a cabo.

\section{Conclusiones: valoración de los resultados obtenidos}

El perfil laboral del agente es el de una mujer joven (entre 25 y 35 años) con una escasa antigüedad en el puesto (menos de tres años), cuyo objetivo es el de desarrollarse profesionalmente e insertarse en el mercado laboral, con un contrato temporal por obra o servicio cuyo puesto depende de una subvención pública procedente del INEM, de la administración autonómica y en mucha menor medida de la administración local. Se deduce de ello una elevada precariedad en el puesto de agente, por el tipo de contratación y por la dependencia del puesto de una subvención o de un programa financiado por las administraciones públicas.

Por otra parte, los procesos de contratación de los agentes han estado dirigidos por el INEM y el grado de autonomía en el puesto depende de la voluntad política y de las posibilidades financieras de la entidad de la cual dependen, al menos hasta que se han producido las transferencias en materia de empleo a las comunidades autónomas, por lo que el modelo INEM está dando paso a un modelo de financiación autonómico, que de momento está siguiendo las mismas pautas que el del INEM (temporalidad del puesto y dependencia financiera y política), excepto en algunas comunidades como la de Galicia y la de Andalucía que están desarrollando un modelo distinto que trata de eliminar la temporalidad del puesto de agente. Precisamente, la temporalidad y la dependencia financiera y política del puesto hace que las expectativas que muestran los agentes sean en la mayoría de los casos negativas, mostrando en unos casos incertidumbre ante el futuro y en otros simplemente desconfianza. 
El perfil laboral del agente expuesto confirma las hipótesis de las que se partía. Sólo en el caso de las comunidades autónomas con un nivel más sólido del desarrollo local se observa una situación algo más favorable en cuanto al tipo de contratación y a la dependencia financiera y política del puesto. Así, en Cataluña y C. Valenciana existen unos niveles algo mayores de agentes con contratos fijos (funcionarios) y en menor medida en Andalucía y Baleares (contratados laborales fijos).

El perfil formativo del agente es el de una persona con experiencia laboral previa, la cual está relacionada con el trabajo de agente y es de gran utilidad para el desempeño del puesto, titulado universitario en Derecho y en menor medida en Empresariales que considera que su formación teórica es la adecuada para el desempeño de su trabajo si bien ha realizado cursos de formación apoyado por la agencia a la cual pertenece y principalmente sobre desarrollo local y en menor medida de informática, gestión de proyectos y economía y empresa, por lo que sus carencias formativas y la necesidad de adquirir nuevos conocimientos son mínimos. De dicho perfil se deduce la necesidad de la formación complementaria del agente, que con un perfil básicamente jurídico, precisa de conocimientos en temas de economía y empresa y, sobre todo, en los temas específicos del Desarrollo Local y de la Gestión de Proyectos.

El perfil formativo descrito presenta matices según comunidades autónomas. Así, el perfil jurídico es más claro en La Rioja, Aragón, Baleares, Castilla-La Mancha, Extremadura y $C$. Valenciana. En cambio, dicho perfil jurídico es compartido con un perfil empresarial en Andalucía y Galicia. Por el contrario, el perfil de empresa es el mayoritario en Castilla-León y Euskadi. En el resto de comunidades se observan otros perfiles como: el territorial (Geografía) en Navarra, Asturias, Cantabria y Cataluña; y el de recursos humanos (Relaciones Laborales, Sociología, Pedagogía) en Canarias, Madrid y Murcia. La relación de las agencias con las universidades en el proceso de formación complementaria de los agentes se da con claridad en Murcia y $C$. Valenciana, mientras que en otras comunidades la formación se lleva a cabo con entidades privadas (Andalucía, Cantabria, Madrid y Euskadi), por parte de la propia entidad a la cual pertenece la agencia (Navarra y Baleares) o por parte de otras entidades sin ánimo de lucro como las Cámaras de Comercio (Aragón) o los sindicatos (Castilla-León).

La gran heterogeneidad de perfiles formativos existentes se explica porque en cada comunidad local existen unas características socioeconómicas específicas que precisan de un profesional con una cualificación apropiada muy concreta, por lo que el perfil de agente estará en función de la preponderancia de unas necesidades determinadas sobre otras. Por otra parte, esto también nos permite afirmar que si 
se quiere consolidar agencias que ofrezcan una perspectiva global del desarrollo local, se hace necesario la creación de equipos multidisciplinares de agentes con capacidades y habilidades muy diversas (empresariales, económicas, jurídicas, de recursos humanos, territoriales, etc.).

Por último, el perfil profesional del agente es el de una persona con un nivel de formalización alto, al realizar una memoria de actividades e informes de evaluación de su trabajo, y con un nivel de colaboración entre agentes también alto aunque con un carácter más informal al ser más habituales los contactos esporádicos y las reuniones puntuales que la pertenencia a grupos de trabajo formales e informales o la pertenencia a asociaciones. En este último caso se ha de señalar que el grado de asociacionismo de los agentes se sitúa en un 30\% aproximadamente a nivel estatal, si bien en determinadas comunidades autónomas es bastante mayor (Navarra, Asturias, Castilla-La Mancha, Castilla-León, Extremadura, Madrid, Euskadi y C. Valenciana). El reconocimiento del trabajo realizado por los agentes es relativamente alto, sobre todo el procedente de los colectivos con los que trabajan y de los directores o responsables de las agencias; si bien, el reconocimiento por parte de la comunidad local en la cual realiza su trabajo el agente es bastante menor.

En definitiva, podemos afirmar que el agente de inserción sociolaboral en España es un profesional del desarrollo local con experiencia, preocupado por adquirir nuevos conocimientos, con un perfil jurídico y de empresa principalmente aunque distinto según comunidades autónomas, a pesar que desempeña su trabajo en una situación de elevada inestabilidad (contratos temporales y supeditación a una subvención temporal), dependencia política (puesto de trabajo coyuntural que depende de las circunstancias del momento y de la voluntad política), bajos salarios y limitado reconocimiento de su trabajo por parte de la comunidad local.

El trabajo del agente de inserción incluye políticas tan diversas como las políticas de educación, formación y aprendizaje continuo para la vida laboral, políticas de asesoramiento y asistencia de acceso al mundo laboral (itinerarios de inserción, autoempleo, ...), políticas de mejora de las infraestructuras locales, políticas de gestión de empresas locales, políticas de apoyo y gestión a la creación de microempresas y de PYMEs y estudios socioeconómicos sobre desarrollo sostenible y políticas medioambientales y de turismo rural y cultural, entre muchas otras más. Por tanto, el papel a desempeñar por el agente es enormemente amplio y heterogéneo, lo cual exige recursos técnicos y materiales considerables y una formación multidisciplinar; a la vez que se trata de un trabajo cuyos resultados se obtienen a largo plazo, requiere de un efecto experiencia importante y de estructuras de apoyo estables y flexibles. 
Sin embargo, de los estudios empíricos realizados hasta el momento y de la opinión de los propios profesionales del desarrollo local se deduce que las condiciones en las que realizan su trabajo los agentes no son las adecuadas para alcanzar los fines mencionados anteriormente. En este sentido, se puede afirmar que se trata de un puesto inestable, con contratos temporales anuales hasta un máximo de cuatro años, no consolidados en el organigrama de las entidades en las cuales realizan su trabajo (principalmente las administraciones locales), dotados de recursos materiales y técnicos insuficientes, dependientes de una subvención pública y sin mecanismos de coordinación de las acciones realizadas. Por otra parte, en ocasiones se observan duplicidades en las estructuras que se ocupan de la inserción sociolaboral, ya que existen diferentes organismos relacionados con ésta sin ningún tipo de coordinación entre ellos. Esto obliga a los agentes, en los casos en los que éstos son capaces, a crear estructuras propias (asociaciones de profesionales o grupos informales de trabajo) a través de las cuales poder llevar a cabo acciones conjuntas, compartir experiencias y ejecutar políticas locales coordinadas. Teniendo en cuenta que los resultados de las políticas locales de empleo no se producen a corto plazo si no que sus consecuencias vienen dadas a largo plazo y requieren también de un proceso largo de ejecución y de seguimiento, se hace necesario contar con plantillas de agentes estables y duraderas; sin embargo, los elevados índices de rotación y de temporalidad que caracterizan las políticas de recursos humanos de las agencias de desarrollo local en España dificultan e impiden el éxito de los planes de acción llevados a cabo en el ámbito del desarrollo local.

Durante los últimos años, se ha reformado parte de la legislación laboral que afecta a los AEDL, en particular, lo que se refiere al límite máximo de cuatro años de contratación subvencionable, lo que mejora, en parte, la inestabilidad laboral de esta figura. Al mismo tiempo, la presión ejercida por Feprodel, abre nuevas expectativas de mejora de las condiciones laborales y profesionales de los agentes en España, si bien las circunstancias y las limitaciones siguen teniendo un elevado nivel de heterogeneidad según comunidades autónomas. 


\section{Bibliografía}

ADELA (1991): Ayuntamientos y desarrollo local: la reactivación de las economías locales, Madrid, ADELA.

AlbURQUerQUe, F. (1997): Metodología para el desarrollo económico local, Santiago de Chile, ILPES-CEPAL.

Allende LANDA, J. (1987): "Desarrollo local y reestructuración urbana-regional", Estudios Territoriales num. 25, pp. 79-97.

ALONSO SANTOS, J. L. y MÉNDEZ, R. (2000): Innovación, pequeña empresa y desarrollo local en España, Madrid, Cívitas.

Antuñano, I. et al. (1993): "Experiencias de desarrollo local en la Comunidad Valenciana: un primer avance", Comunicación $2^{\circ}$ Congreso de Economía Valenciana, 28-30 abril, Castellón, Instituto Valenciano de Investigaciones Económicas IVIE.

Aprodel (2004): Encuesta sobre la situación actual del Desarrollo Local en la Comunidad de Madrid, Informe Aprodel, Asociación de Profesionales del Desarrollo Local, Madrid. Trabajo no publicado pero disponible en: www.aprodelmadrid.org.

Becattini, G., Costa Campí, M. T. y Trullen, J. (2002): Desarrollo local: teorías y estrategias, Madrid, Cívitas.

Cachón Rodríguez, L. (1999): "sobre desarrollo local y nuevos yacimientos de empleo", Política y Sociedad núm. 31, pp. 117-130.

Calvo Palomares, R. (2008): "La situación laboral-contractual del agente de desarrollo local como factor limitador de los efectos de su actividad: una propuesta de clasificación", Temas laborales: revista andauza de trabajo y bienestar social, núm. 93, pp. 157-172.

Calvo Palomares, R. (2009): "Reflexiones sobre el modelo de desarrollo local: propuesta de un decálogo de retos de futuro", Investigaciones regionales, núm. 14 , pp. 133-153.

CAMAGNI, R. (2003): "Incertidumbre, capital social y desarrollo local: enseñanzas para un gobernabilidad sostenible del territorio", Investigaciones Regionales núm. 2, primavera, pp. 31-58. 
Campos, V., Cantarero, S. y Sanchis, J. R. (2002): "Un estudio sobre el perfil y las características del agente de desarrollo local en la Comunidad Valenciana. Análisis comparativo con el resto de España", Revista Valenciana de Economía y Hacienda núm. 5, pp. 147-186.

Contell García, T. (1996): "Empresarialidad y emprenditorialidad. Dos conceptos claves para el desarrollo local", Revista Valenciana de Estudios Autonómicos núm. 21 , pp. $51-70$.

Costa CAmpí, M. T. et al. (1993): EXCEL. Cooperación entre empresas y sistemas productivos locales, Madrid, Edita IMPI.

CRUz Roche, P. (1984): "Problemática empresarial en un proceso de desarrollo local", Información Comercial Española núm. 611, pp. 141-146.

De Pablo Valenciano, J., y URIBe Toril, J. (2009): “Emprendimiento de la economía social y desarrollo local: la promoción de incubadoras de empresas de economía social en Andalucía", Revista CIRIEC-España núm. 64, abril, pp. 5-33.

FOREM (2000): Estudio de necesidades de formación de agentes de desarrollo local y figuras profesionales afines, Secretaría de Formación y Cultura de la C.S. de CC.OO., Madrid, Edita Fundación Formación y Empleo Miguel Escalera.

GAzÓN, J. (1996): "El desarrollo local y regional basado en las iniciativas PYME. Metodología de SGP Consultants", Economía Industrial núm. 309, pp.127-138.

Giner Mansó, Y., Muriel de los Reyes, M. J. y Toledano Redondo, F. J. (1999): "El empresario y la creación de nuevas empresas en el ámbito rural: impacto de las políticas comunitarias", Revista Europea de Dirección y Economía de la Empresa Vol. 8, núm. 3, pp. 185-198.

Gonzalez CARDonA, J. (2007): "El agente de empleo y desarrollo local: competencias profesionales y trabajo en red". En, ROSAT ACED, J.I. (coordinador): Guía práctica de gestión de personal y recursos humanos en la administración local, Edita Aranzazi, pp. 247-260.

HONRUBIA LóPEZ, J. (coordinador) (2004): Globalización y desarrollo local: una perspectiva valenciana, Valencia, Edita Universitat de València.

MANRIQUe DE LARA, V. (2008): "La administración pública local como agente de empleo: el caso de la Diputación de Granada". En, De Pablos RamireZ, J.C. (coor- 
dinador): El empleo de cerca: estudios sobre agentes y estrucutras en la provincia de Granada, Granada, Edita Universidad de Granada, pp. 91-108.

Martínez Puche, A. et al. (2000): Herramientas para el desarrollo local, Alicante, Edita CEDER-Aitana.

Medina Hernández, U., Rodríguez Ferrer, T. y Fuentes Medina, M. L. (1996): "Agencias de desarrollo local. El caso de Canarias", Revista CIRIEC-España núm. 24, noviembre, pp. 40-62.

Mozas Moral, A., y Bernal Jurado, E. (2006): "Desarrollo territorial y economía social", Revista CIRIEC-España núm. 55, noviembre, pp. 125-140.

OCDE (1999): Redes de empresas y desarrollo local: competencia y cooperación en los sistemas productivos locales, París, Organización de Cooperación y Desarrollo Económico.

Orero, J. I. (1993): "Promoción económica local en la Comunidad Valenciana", Comunicación $2^{\circ}$ Congreso de Economía Valenciana, 28-30 abril, Castellón, Instituto Valenciano de Investigaciones Económicas, IVIE.

Pérez Ramírez, B. y Carrillo Benito, E. (2001): Desarrollo local: manual de uso, Madrid, Editorial ESIC.

Rodríguez Alvarez, J. M. (coordinador) (2001): Experiencias prácticas de desarrollo local: 19 estudios de casos de dinamización socioeconómica en ámbitos locales, Barcelona, Edita Bayer Hnos.

Rodríguez GutiérRez, F. (1999): Manual de desarrollo local, Gijón, Trea.

SANCHIS, J. R. (1999): "Las estrategias de desarrollo local: aproximación metodológica desde una perspectiva socioeconómica e integral", Revista de Dirección, Organización y Administración de Empresas núm. 21, enero, pp. 147-160.

SANCHIS, J. R. (2001): "Creación y consolidación de empresas mediante el crecimiento en red: su aplicación al desarrollo local", Revista de Dirección, Organización y Administración de Empresas núm. 25, febrero, pp. 14-25.

SANCHIS, J. R. (2004): "Inserción sociolaboral, economía social y desarrollo local: el papel del Agente de Empleo y Desarrollo Local en las políticas locales de inser- 
ción sociolaboral", Noticias de la Economía pública, social y cooperativa núm. 41, pp. 73-77.

SANCHIS, J. R. (2006): El papel del agente de empleo y desarrollo local en la implementación de las políticas locales de empleo y en la creación de empresas en España: estudio empírico y análisis comparativo entre CCAA, Ministerio de Trabajo y Asuntos Sociales, Madrid.

SANCHIS, J. R. y CAMPOS, V. (2002): "El agente de desarrollo local y las políticas activas de empleo. Estudio empírico aplicado a la Comunidad Valenciana y su comparación con el resto de España", Arxius de Ciències Socials núm. 7, novembre, pp. 111-133.

SANCHIS, J. R. y CAMPOS, V. (2005): "Inserción sociolaboral, economía social y desarrollo local. Estudio empírico sobre la realidad actual del Agente de Empleo y Desarrollo Local en España", Revista CIRIEC-España, núm. 52, agosto, pp. 279-306.

SanCHIS, J. R. y Cantarero, S. (1999): "El agente de fomento como instrumento de creación y desarrollo de empresas", Revista Europea de Dirección y Economía de la Empresa, Volumen 8 núm. 3, pp. 163-184.

Sanchis, J. R., CAntarero, S. y Campos, V. (2003a): El Agente de Desarrollo Local en la creación y desarrollo de empresas, Sevilla, Editorial D+D digital.

Sanchis, J. R., Cantarero, S. y Campos, V. (2003b): "El agente de desarrollo local y su papel en la creación y desarrollo de empresas. Estudio empírico aplicado a la Comunidad Valenciana", en Rubert, J.J. y Fuertes (eds.): La economía regional en el marco de la nueva economía, pp. 99-122.

SOlA SOlA, J. y PelICER, P. (1992): "La cooperación PYMEs-grandes empresas y su incidencia en el desarrollo local", Economía Industrial núm. 286, pp. 43-47.

Tomas CARPI, J.A. (2008): "El Desarrollo Local sostenible en clave estratégica", Revista CIRIEC-España, núm. 61, agosto, pp. 73-101.

Vázquez Barquero, A. (1993): Política económica local, Madrid, Pirámide.

VÁzquez Barquero, A. (1996a): "La política de desarrollo económico local en Europa", Revista Asturiana de Economía núm. 5, pp. 7-25. 
VÁzQuez Barquero, A. (1996b): "Desarrollo local y disparidades regionales en España", Papeles de Economía Española núm. 67, pp. 81-95.

VÁZquez BARQUeRO, A. (1998): "Desarrollo endógeno: mecanismos institucionales y culturales", Revista Valenciana d'Estudis Autonòmics núm. 21, pp. 71-92.

VÁzquez BARQuero, A. y Garofoll, G. (editores) (1995): Desarrollo Económico Local en Europa, Madrid, Colegio de Economistas de Madrid. 


\section{ANEXO}

TABLA 3. Perfil laboral del agente: denominación, sexo, edad, antigüedad y motivación

\begin{tabular}{|c|c|c|c|c|c|c|c|c|c|}
\hline Denominación & $\%$ & Sexo & $\%$ & Edad & $\%$ & Antigijedad & $\%$ & Motivación* & $\%$ \\
\hline AEDL & 54,3 & Masculino & 35,6 & Menos de 25 & 1,3 & 1 año & 17,2 & Desempleo & 38,5 \\
\hline ADL & 10,0 & Femenino & 64,4 & Entre 25 y 30 & 30,1 & 2 años & 23,6 & $\begin{array}{l}\text { Experiencia } \\
\text { anterior }\end{array}$ & 34,6 \\
\hline ADR & 1,7 & TOTAL & 100 & Entre 31 y 35 & 37,6 & 3 años & 19,7 & $\begin{array}{l}\text { Motivos } \\
\text { personales }\end{array}$ & 57,3 \\
\hline Técnico local & 12,9 & & & Entre 36 y 40 & 18,5 & 4 años & 11,7 & Otros motivos & 4,8 \\
\hline $\begin{array}{l}\text { Promotor } \\
\text { empleo }\end{array}$ & 12,3 & & & Entre 41 y 45 & 8,7 & 5 años & 10,3 & & \\
\hline Gerente/ Dtor. & 6,2 & & & Más de 45 & 3,8 & Más de 5 & 17,5 & & \\
\hline Otra & 2,6 & & & TOTA & 100 & TOTAL & 100 & & \\
\hline TOTAL & 100 & & & IVIAL & 100 & Media & 3,7 & & \\
\hline
\end{tabular}

* Respuesta múltiple. Fuente: elaboración propia

TABLA 4. Perfil laboral del agente: contratación

\begin{tabular}{|c|c|c|c|c|c|c|c|c|c|}
\hline Contrato & $\%$ & Subvención & $\%$ & $\begin{array}{c}\text { Entidad } \\
\text { financiadora* }\end{array}$ & $\%$ & $\begin{array}{c}\text { Entidad } \\
\text { contratante }\end{array}$ & $\%$ & $\begin{array}{l}\text { Proceso de } \\
\text { selección* }\end{array}$ & $\%$ \\
\hline Funcionario & 5,6 & Sí & 89,4 & Ayuntamiento & 17,4 & Ayuntamiento & 56,4 & Valorar C.V. & 84,6 \\
\hline Laboral & 5,4 & No & 10,6 & Diputación & 5,4 & Diputación & 9,6 & Entrevista & 83,3 \\
\hline $\begin{array}{l}\text { Laboral } \\
\text { temporal }\end{array}$ & 34,4 & \multirow{5}{*}{ TOTAL } & \multirow{5}{*}{100} & Consejería & 71,8 & Consejería & 8,6 & Pruebas & 30,3 \\
\hline $\begin{array}{l}\text { Obras } 0 \\
\text { servicio }\end{array}$ & 54,0 & & & INEM & 23,5 & INEM & 8,0 & \multirow{4}{*}{ Proyecto } & \multirow{4}{*}{28,0} \\
\hline Prácticas & 0,2 & & & Empresa Privada & 0,5 & Mancomunidad & 13,2 & & \\
\hline Otro & 0,4 & & & Mancomunidad & 1,8 & Otra & 4,2 & & \\
\hline TOTAL & 100 & & & Otra & 0,3 & TOTAL & 100 & & \\
\hline
\end{tabular}

* Respuesta múltiple. Fuente: elaboración propia 


\section{TABLA 5. Perfil laboral del agente: autonomía en el trabajo y expectativas futuras \%}

\begin{tabular}{|l|c|c|c|l|c|l|l|l|l|}
\hline $\begin{array}{c}\text { Grado auto- } \\
\text { nomía en: }\end{array}$ & $\begin{array}{c}\text { Fijación } \\
\text { objetivos }\end{array}$ & $\begin{array}{c}\text { Fijación } \\
\text { acciones }\end{array}$ & $\begin{array}{l}\text { Ejecución } \\
\text { acciones }\end{array}$ & $\begin{array}{l}\text { Factores* } \\
\text { autonomía }\end{array}$ & $\%$ & Expectativas & $\%$ & $\begin{array}{l}\text { De qué } \\
\text { dependen* }\end{array}$ & $\%$ \\
\hline No tiene & 8,7 & 5,8 & 2,9 & Antigüedad & 15,7 & Consolidar & 39,8 & $\begin{array}{l}\text { Decisión } \\
\text { políitca }\end{array}$ & 65,5 \\
\hline Parcial & 66,4 & 71,5 & 55,3 & Jerarquía & 27,5 & Desconoce & 47,5 & $\begin{array}{l}\text { Alcanzar } \\
\text { Rdos }\end{array}$ & 26,9 \\
\hline Total & 24,9 & 22,7 & 41,8 & Trabajo & 70,6 & Abandonar & 12,7 & Subvención & 63,2 \\
\hline TOTAL & 100 & 100 & 100 & Otros & 7,4 & TOTAL & 100 & Otros & 2,1 \\
\hline
\end{tabular}

* Respuesta múltiple. Fuente: elaboración propia

TABLA 6. Nivel de satisfacción en diversos aspectos del trabajo del agente (en porcentaje)

\begin{tabular}{|l|c|c|c|c|c|c|c|}
\hline $\begin{array}{c}\text { Nivel de } \\
\text { satisfacción }\end{array}$ & $\begin{array}{c}\text { Funciones } \\
\text { realizadas }\end{array}$ & $\begin{array}{c}\text { Responsabi- } \\
\text { lidad ejercida }\end{array}$ & Salario & $\begin{array}{c}\text { Ambiente } \\
\text { laboral }\end{array}$ & $\begin{array}{c}\text { Perspectivas } \\
\text { futuro }\end{array}$ & $\begin{array}{c}\text { Resultados } \\
\text { obtenidos }\end{array}$ & $\begin{array}{c}\text { Reconocimiento } \\
\text { social }\end{array}$ \\
\hline 1 (mínima) & 1,0 & 1,5 & 5,0 & 2,4 & 19,4 & 1,5 & 2,2 \\
\hline 2 & 5,9 & 5,9 & 15,8 & 6,5 & 24,2 & 4,4 & 15,3 \\
\hline 3 & 19,7 & 24,4 & 31,1 & 19,5 & 30,0 & 25,6 & 33,6 \\
\hline 4 & 48,1 & 45,8 & 36,6 & 33,4 & 21,4 & 54,4 & 38,0 \\
\hline 5 (máxima) & 25,3 & 22,4 & 11,5 & 38,2 & 5,0 & 14,1 & 10,9 \\
\hline TOTAL & 100,00 & 100,00 & 100,00 & 100,00 & 100,00 & 100,00 & 100,00 \\
\hline
\end{tabular}

Fuente: elaboración propia

TABLA 7. Perfil formativo del agente: experiencia previa

\begin{tabular}{|c|c|c|c|c|c|c|c|}
\hline $\begin{array}{c}\text { Trabajar como } \\
\text { agente }\end{array}$ & $\%$ & $\begin{array}{c}\text { Experiencia } \\
\text { laboral }\end{array}$ & $\%$ & $\begin{array}{c}\text { Relacionada } \\
\text { con su trabajo }\end{array}$ & $\%$ & $\begin{array}{c}\text { Utilidad de } \\
\text { experiencia }\end{array}$ & $\%$ \\
\hline Sí & 33,9 & Sí & 77,6 & Sí & 58,5 & Sí & 89,6 \\
\hline No & 66,1 & No & 22,4 & No & 41,5 & No & 10,4 \\
\hline TOTAL & 100 & TOTAL & 100 & TOTAL & 100 & TOTAL & 100 \\
\hline
\end{tabular}

Fuente: elaboración propia 
TABLA 8. Perfil formativo del agente: formación teórica

\begin{tabular}{|c|c|c|c|c|c|c|c|}
\hline Titulación & $\%$ & $\begin{array}{l}\text { Formación } \\
\text { adecuada }\end{array}$ & $\%$ & $\begin{array}{l}\text { Fomento } \\
\text { agencia }\end{array}$ & $\%$ & Cursos & $\%$ \\
\hline No universitarios & 0,8 & Sí & 77,9 & Sí & 84,6 & Sí & 93,1 \\
\hline Licenciatura de Derecho & 20,3 & No & 22,1 & No & 15,4 & No & 6,9 \\
\hline $\begin{array}{l}\text { Licenciatura Empresariales/ } \\
\text { ADE }\end{array}$ & 16,9 & \multirow{19}{*}{ TOTAL } & \multirow{19}{*}{100} & \multirow{19}{*}{ TOTAL } & \multirow{19}{*}{100} & \multirow{19}{*}{ TOTAL } & \multirow{19}{*}{100} \\
\hline $\begin{array}{l}\text { Diplomatura Relaciones } \\
\text { Laborales }\end{array}$ & 10,5 & & & & & & \\
\hline Licenciatura de Economía & 10,3 & & & & & & \\
\hline $\begin{array}{l}\text { Licenciatura Geografía e } \\
\text { Historia }\end{array}$ & 8,8 & & & & & & \\
\hline Diplomatura de Empresariales & 6,1 & & & & & & \\
\hline Licenciatura de Pedagogía & 3,8 & & & & & & \\
\hline Licenciatura de Psicología & 3,6 & & & & & & \\
\hline Diplomatura de Trabajo Social & 3,3 & & & & & & \\
\hline Licenciatura de Sociología & 2,9 & & & & & & \\
\hline Ingeniería Técnica Agrícola & 2,1 & & & & & & \\
\hline Licenciatura de Filología & 1,9 & & & & & & \\
\hline Licenciatura de Biología & 1,7 & & & & & & \\
\hline Licenciatura Ciencias Políticas & 1,5 & & & & & & \\
\hline Diplomatura de Magisterio & 1,5 & & & & & & \\
\hline Diplomatura de Turismo & 0,8 & & & & & & \\
\hline Licenciatura de Periodismo & 0,4 & & & & & & \\
\hline Diplomatura de Documentación & 0,4 & & & & & & \\
\hline Otras titulaciones & 2,4 & & & & & & \\
\hline TOTAL & 100 & & & & & & \\
\hline
\end{tabular}

Fuente: elaboración propia 
TABLA 9. Perfil formativo del agente: formación complementaria

\begin{tabular}{|c|c|c|c|}
\hline Cursos realizados* & $\%$ & Entidad formadora* & $\%$ \\
\hline Desarrollo Local & 75,6 & La propia entidad & 28,3 \\
\hline Economía y Empresa & 48,1 & INEM & 19,3 \\
\hline Gestión de proyectos & 49,0 & UNED & 11,9 \\
\hline Informática & 52,2 & Universidad & 33,9 \\
\hline Gestión de RR.HH. & 22,1 & Entidad privada & 27,6 \\
\hline Turismo & 2,9 & Consejería & 14,8 \\
\hline Unión Europea & 2,0 & Diputación Provincial & 16,8 \\
\hline Orientación laboral & 3,1 & FEMP & 7,6 \\
\hline Inserción sociolaboral & 2,9 & Cámara de Comercio & 2,2 \\
\hline Prevención riesgos laborales & 1,3 & Sindicatos & 3,6 \\
\hline lgualdad & 1,1 & \multirow{3}{*}{ Otras entidades } & \multirow{3}{*}{28,1} \\
\hline Idiomas & 1,8 & & \\
\hline Otros cursos & 15,7 & & \\
\hline
\end{tabular}

* Respuesta múltiple. Fuente: elaboración propia

TABLA 10. Carencias formativas y de conocimientos para el desempeño del puesto (\%)

\begin{tabular}{|l|c|c|c|c|c|c|c|}
\hline \multicolumn{7}{|c|}{ Carencias formativas de la titulación académica } \\
\hline Carencia & Economía & Empresa & Derecho & $\begin{array}{c}\text { Temas } \\
\text { sociales }\end{array}$ & $\begin{array}{c}\text { Gestión } \\
\text { RR.HH. }\end{array}$ & $\begin{array}{c}\text { Gestión } \\
\text { Proyectos }\end{array}$ & Informática \\
\hline NS/NC & 1,0 & 1,5 & 1,7 & 2,5 & 2,3 & 1,2 & 1,9 \\
\hline 1 (total) & 5,1 & 1,9 & 9,1 & 3,0 & 3,6 & 1,7 & 1,7 \\
\hline 2 & 23,0 & 16,2 & 25,9 & 16,2 & 15,6 & 9,5 & 6,3 \\
\hline 3 & 32,5 & 33,8 & 28,9 & 30,2 & 35,4 & 30,8 & 32,3 \\
\hline 4 & 24,5 & 32,7 & 20,7 & 34,8 & 32,3 & 40,3 & 42,0 \\
\hline 5 (nula) & 13,9 & 13,9 & 13,7 & 13,3 & 10,8 & 16,5 & 15,8 \\
\hline TOTAL & 100,00 & 100,00 & 100,00 & 100,00 & 100,00 & 100,00 & 100,00 \\
\hline
\end{tabular}




\begin{tabular}{|l|c|c|c|c|c|c|c|}
\hline \multicolumn{7}{|c|}{ Necesidad de conocimientos para el desempeño del puesto } \\
\hline Necesidad & $\begin{array}{c}\text { Realidad } \\
\text { Socioecon. }\end{array}$ & $\begin{array}{c}\text { Mercado } \\
\text { Trabajo }\end{array}$ & $\begin{array}{c}\text { Economía } \\
\text { Empresa }\end{array}$ & $\begin{array}{c}\text { Legislación } \\
\text { actual }\end{array}$ & $\begin{array}{c}\text { Gestión } \\
\text { Proyectos }\end{array}$ & $\begin{array}{c}\text { Gestión } \\
\text { RR.HH. }\end{array}$ & Informática \\
\hline NS/NC & 0,0 & 0,0 & 0,9 & 0,8 & 0,9 & 1,1 & 0,5 \\
\hline 1 (total) & 0,0 & 0,6 & 2,1 & 0,2 & 0,0 & 1,5 & 0,6 \\
\hline 2 & 0,4 & 0,8 & 2,9 & 1,7 & 1,0 & 10,1 & 2,1 \\
\hline 3 & 2,4 & 11,8 & 28,2 & 19,4 & 7,4 & 38,1 & 24,8 \\
\hline 4 & 20,4 & 44,1 & 43,8 & 39,6 & 37,9 & 34,5 & 40,8 \\
\hline 5 (nula) & 76,8 & 42,7 & 22,1 & 38,3 & 52,8 & 14,7 & 31,2 \\
\hline TOTAL & 100,00 & 100,00 & 100,00 & 100,00 & 100,00 & 100,00 & 100,00 \\
\hline
\end{tabular}

Fuente: elaboración propia

TABLA I I. Perfil profesional del agente: características necesarias en su trabajo (\%)

\begin{tabular}{|c|c|c|c|c|c|c|c|c|}
\hline $\begin{array}{c}\text { Importan- } \\
\text { cia }\end{array}$ & $\begin{array}{c}\text { Seleccion } \\
\text { informac }\end{array}$ & $\begin{array}{c}\text { Capacidad } \\
\text { Comunicac. }\end{array}$ & $\begin{array}{c}\text { Visión } \\
\text { general }\end{array}$ & $\begin{array}{c}\text { Análisis y } \\
\text { sintesis }\end{array}$ & $\begin{array}{c}\text { Imaginación } \\
\text { y creatividad }\end{array}$ & $\begin{array}{c}\text { Decisión y } \\
\text { ejecución }\end{array}$ & Gestión & $\begin{array}{c}\text { Negocia- } \\
\text { ción }\end{array}$ \\
\hline \begin{tabular}{c} 
(mínima) \\
\hline 2
\end{tabular} & 0,4 & 0,0 & 0,3 & 0,4 & 0,2 & 0,6 & 0,8 & 0,8 \\
\hline 3 & 10,3 & 4,4 & 9,2 & 15,9 & 17,0 & 13,8 & 9,6 & 20,8 \\
\hline 4 & 45,3 & 33,2 & 46,3 & 49,1 & 36,5 & 47,0 & 44,3 & 43,2 \\
\hline 5 & 43,4 & 62,2 & 43,4 & 34,2 & 44,2 & 36,9 & 44,5 & 31,6 \\
\hline (máxima) & 0,8 & 0,4 & 2,1 & 1,7 & 0,8 & 3,6 \\
\hline TOTAL & 100,00 & 100,00 & 100,00 & 100,00 & 100,00 & 100,00 & 100,00 & 100,00 \\
\hline
\end{tabular}

Fuente: elaboración propia 
TABLA I2. Perfil profesional del agente: mecanismos de control y relaciones

\begin{tabular}{|c|c|c|c|c|c|c|c|}
\hline $\begin{array}{l}\text { Memoria de } \\
\text { actividades }\end{array}$ & $\%$ & $\begin{array}{l}\text { Evaluación } \\
\text { del trabajo }\end{array}$ & $\%$ & $\begin{array}{l}\text { Relaciones con } \\
\text { otros agentes }\end{array}$ & $\%$ & $\begin{array}{l}\text { Tipos de } \\
\text { relaciones* }\end{array}$ & $\%$ \\
\hline Sí & 98,8 & Sí & 83,8 & Sí & 97,9 & Asociación & 31,1 \\
\hline No & 1,2 & No & 16,2 & No & 2,1 & Grupo formal & 32,2 \\
\hline \multirow{4}{*}{ TOTAL } & \multirow{4}{*}{100} & \multirow{4}{*}{ TOTAL } & \multirow{4}{*}{100} & \multirow{4}{*}{ TOTAL } & \multirow{4}{*}{100} & Grupo informal & 24,7 \\
\hline & & & & & & Reuniones & 72,2 \\
\hline & & & & & & Contactos & 87,9 \\
\hline & & & & & & Otras relaciones & 4,3 \\
\hline
\end{tabular}

* Respuesta múltiple. Fuente: elaboración propia

TABLA I3. Reconocimiento del trabajo del agente (\%)

\begin{tabular}{|c|c|c|c|c|}
\hline Valoración & Director agencia & Políticos & Colectivos & Comunidad local \\
\hline NS/NC & 20,3 & 0,4 & 1,2 & 1,3 \\
\hline 1 (mínima) & 0,9 & 2,6 & 0,4 & 2,3 \\
\hline 2 & 4,5 & 12,1 & 1,9 & 11,3 \\
\hline 3 & 13,9 & 30,4 & 25,5 & 37,9 \\
\hline 4 & 37,3 & 43,2 & 51,9 & 39,8 \\
\hline 5 (máxima) & 23,1 & 11,3 & 19,1 & 7,4 \\
\hline TOTAL & 100,00 & 100,00 & 100,00 & 100,00 \\
\hline
\end{tabular}

Fuente: elaboración propia 